Review

\title{
Use of Complementary and Alternative Medicine for Children with Sickle Cell Disease: Prevalence and Factors Associated with Use
}

Mohammed Alsabri ${ }^{1}$, Kusum Viswanathan ${ }^{1,}{ }^{*}$, Fiorella Castillo ${ }^{1}$, Paridhi Ghai ${ }^{2}$, Maryam Hemmat Hamrah ${ }^{2}$

1. Brookdale University Hospital and Medical center 1Brookdale Plaza, Brooklyn, NY 11212, USA; E-Mails: malsabri@bhmcny.org; kviswana@bhmcny.org; ficastil@bhmcny.org

2. Saba University School of Medicine, Church street, The Bottom, Caribbean Netherlands; EMails: p.ghai@saba.edu; $\underline{\text { m.hamrah@saba.edu }}$

* Correspondence: Kusum Viswanathan; E-Mail: kviswana@bhmcny.org

Academic Editor: Gerhard Litscher

OBM Integrative and Complementary Medicine

2021, volume 6 , issue 2

doi:10.21926/obm.icm.2102013
Received: December 26, 2020

Accepted: March 25, 2021

Published: April 17, 2021

\begin{abstract}
Sickle cell disease (SCD) is one of the most common hematologic disorders in the world. In the United States, it is the single most common genetic hematologic disorder. The burden of SCD and its complications on pediatric patients is tremendous, especially acute vasoocclusive pain crises. Although allopathic medicine has provided numerous pain medications to treat this, they do not come without side effects and are not always effective. This review explores complementary and alternative medicine (CAM) methods of pain management available for pediatric SCD patients. A PubMed literature search was conducted, and the reference lists of shortlisted studies and review articles were screened after application of the exclusion criteria; 14 articles were used in this literature analysis. A wide range of CAM therapies exist. Common ones include praying, spirituality, and massage. Factors associated with CAM use are parental use of CAM, gender, SCD/pain severity, patient's age, caregiver's education level, and in some cases, financial means to access CAM methods. CAM has shown to be effective in reducing pain, patients' depression and anxiety, analgesic use, and in increasing daily functioning. Thus, CAM has demonstrated potential in reducing pain and improving the quality of life of pediatric SCD patients. It holds great promise to be regularly
\end{abstract}

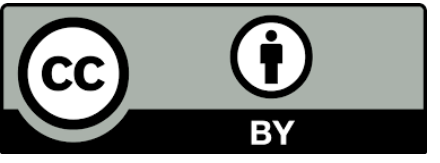

(C) 2021 by the author. This is an open access article distributed under the conditions of the Creative Commons by Attribution License, which permits unrestricted use, distribution, and reproduction in any medium or format, provided the original work is correctly cited. 
encouraged by providers and employed by patients. Further research is needed on a larger scale to better study its efficacy; however, CAM use has repeatedly been associated with improved functioning and pain control.

\section{Keywords}

Sickle cell disease; pediatrics; pain; complementary and alternative medicine

\section{Background}

Sickle cell disease (SCD) is a life-threatening condition that affects millions of people worldwide. In 2010, SCD was recognized by the World health organization and the United Nations as a global health problem that is expected to increase in the future [1]. In the United States, one out of every 375 African Americans is affected by SCD, making SCD the most common genetic hematologic disorder in the United States [2]. The hallmark of SCD is pain and chronic anemia. In addition, SCD has many clinical complications including but not limited to cerebrovascular accidents (CVA), acute chest syndrome (ACS), gallstones, and sepsis [2, 3]. Most, if not all, complications are accompanied by pain and the majority of sickle cell patients live with pain regularly, the management of which can be a daunting task [3]. The pain could be acute or chronic. Acute pain (often referred to as pain crises) is the single most debilitating aspect of $\operatorname{SCD}[2,3]$. This crisis is usually a result of the occlusion of blood vessels by sickled red blood cells adhering to white cells and platelets and the endothelial cells $[2,3]$. The pain is excruciating and starts suddenly in the bones particularly the back and extremities [2, 3]. Although dehydration, infection, and exposure to cold are some of the triggers, often there may not be a clear-cut trigger [2-4]. It is not uncommon for many patients with SCD to also experience chronic pain. Chronic pain, as defined by the American Psychological Association, is "pain that lasts longer than six months and affects how a person lives their daily life". The mechanisms of chronic pain in SCD remain unclear, often caused by orthopedic problems (avascular necrosis, vertebral collapse, chronic arthritis), however, the causes are most likely to be multifactorial $[2,3]$. A study by Ballas et al. showed that SCD pain crises were the most common cause of hospitalizations, and these were associated with anxiety, fear, and depression. In addition, more than half of hospital admissions for acute painful episodes were readmissions within one month of a prior discharge [4].

Several pharmacologic medications have been tried in order to control SCD pain, with opioids being the most conventional treatment. However, uncontrolled persistent pain often remains a difficult clinical problem, particularly in older patients. Patients with chronic pain are treated with opioids but can come to emergency rooms with breakthrough acute pain. Undertreatment of pain in SCD is a common problem in emergency rooms. Over the last decade, progress has been made in accelerating SCD research and creating additional treatment modalities including complementary and alternative medicine (CAM) therapies for the treatment of SCD. According to the National Center for Complementary and Integrative Health (NCCIH), "CAM is a group of diverse medical and health care systems, practices, and products that are not generally considered part of conventional medicine". Its use has had an upward trend amongst the sickle cell pediatric population. There is currently a wide range of CAM therapies that SCD patients employ for a 
variety of reasons. These include anything from meditation and spirituality to yoga, behavioral and cognitive strategies, and biofeedback-assisted relaxation [5-7]. However, there is a shortage of research studies on the effectiveness of CAM use among pediatric sickle cell patients, and the factors that are associated with its use. The objective of this review is to describe the prevalence of CAM, as well as the factors associated with CAM use in pediatric SCD.

\section{Methods}

The articles reviewed in this literature analysis were obtained from the PubMed Database from which the most recent search was conducted on February 2021. The keywords/Mesh terms used for the search strategy were: ((sickle cell) AND pediatrics) AND pain) AND complementary medicine) Sort by: Most Recent ("sickle"[All Fields] OR "sickled"[All Fields] OR "sickles"[All Fields] OR "sickling"[All Fields]) AND ("cells"[MeSH Terms] OR "cells"[All Fields] OR "cell"[All Fields]) AND ("paediatrics"[All Fields] OR "pediatrics"[MeSH Terms] OR "pediatrics"[All Fields] OR "paediatric"[All Fields] OR "pediatric"[All Fields]) AND ("pain"[MeSH Terms] OR "pain"[All Fields]) AND ("complementary therapies"[MeSH Terms] OR ("complementary"[All Fields] AND "therapies"[All Fields]) OR "complementary therapies"[All Fields] OR ("complementary"[All Fields] AND "medicine"[All Fields]) OR "complementary medicine"[All Fields]) Translations sickle: "sickle"[All Fields] OR "sickled"[All Fields] OR "sickles"[All Fields] OR "sickling"[All Fields] cell: "cells"[MeSH Terms] OR "cells"[All Fields] OR "cell"[All Fields] pediatrics: "paediatrics"[All Fields] OR "pediatrics"[MeSH Terms] OR "pediatrics"[All Fields] OR "paediatric"[All Fields] OR "pediatric"[All Fields] pain: "pain"[MeSH Terms] OR "pain"[All Fields] complementary medicine: "complementary therapies"[MeSH Terms] OR ("complementary"[All Fields] AND "therapies"[All Fields]) OR "complementary therapies"[All Fields] OR ("complementary"[All Fields] AND "medicine"[All Fields]) OR "complementary medicine"[All Fields]. This search produced 411 results.

\subsection{Inclusion Criteria}

Primary articles that evaluated the prevalence of CAM, the effectiveness of CAM, and factors associated with the use of CAM in pediatric SCD. Reference lists and bibliographies of eligible peer-reviewed articles also were searched for relevant material.

\subsection{Exclusion Criteria}

Secondary or tertiary articles, including review articles and meta-analyses. Control trials involving animal subjects, case series, articles that were published before 2000, and non-English literature were excluded. Studies focused on SCD in the adult population (age above 21 years) were also excluded.

After applying these exclusion criteria, 14 articles were used in this literature analysis.

\section{Results}

The 14 selected studies were carefully examined. Their results and data were analyzed and stratified based on our aim. These studies demonstrated various experimental, observational, and 
cross-sectional methods to report the existence, prevalence of use, and effectiveness of a variety of CAM methods in SCD patients. Below are the findings of our investigations.

\subsection{Prevalence of Using CAM among Pediatric SCD Patients}

There are many types of CAM, Stinson et al. [8] classified CAM interventions into three categories: physical (e.g., heat massage, acupuncture), behavioral (e.g., biofeedback, daily coping skills), and psychologic (e.g., prayers, guided imagery, virtual reality) modalities [8]. There is a range of CAM therapies that SCD patients employ. These include anywhere from meditation and spirituality, to yoga and biofeedback-assisted relaxation [5-7]. The most common CAM methods appear to be prayer and meditation, cognitive behavioral therapy, biofeedback, relaxation techniques, acupuncture, and hypnosis [3] (Table 1). However, deep breathing, distraction, positive self-talk, sleeping, napping, watching television (TV), reading, taking a hot bath, and using heating pads are also employed by many patients [5-7].

Table 1 Common methods of CAM employed by SCD pediatric patients.

\begin{tabular}{l}
\hline Most common CAM methods \\
\hline Prayer \\
Meditation/Spirituality \\
Relaxation \\
Sleeping/napping \\
Heat application \\
Watching TV/distraction \\
Deep breathing \\
\hline Lesser common CAM methods \\
\hline Acupuncture \\
Hypnosis \\
Exercise \\
Biochemical remedies (herbs/vitamins) \\
\hline
\end{tabular}

Sanchez et al. [9] found that 63\% of their study participants used CAM overall for SCD pain and that the three most common types of CAM used were prayer (60\%), vitamins/minerals (10\%), and massage (7.5\%). In this study, the percent of non-prayer CAM use in their participants was $23 \%$ [9]. However, they go further to report that $50 \%$ of caregivers of SCD children had not previously heard about CAM, and most participants who did not use CAM did not do so because of a lack of information rather than lack of interest [9]. More specifically, Sibinga et al. [10] report that $54 \%$ of their study participants used CAM methods for their SCD child prior to the study. Of those, $42 \%$ used prayer, $11 \%$ used spiritual healing, and/or $2 \%$ used energy healing [10]. Twenty-eight percent used lifestyle/mind-body CAM therapies such as relaxation techniques, exercise, diet, and/or imagery [10]. Twelve percent used biochemical CAM therapies: herbs, megavitamin therapy, and/or folk remedies, and finally, 5\% used massage [10]. Many parents of SCD children were 
interested in CAM, 83\% of respondents felt CAM could be helpful and $60 \%$ reported interest in trying other CAM methods [10]. Furthermore, Yoon et al. [11] who investigated comprehensive techniques of pain management for pediatric SCD patients found that more than $70 \%$ of caregivers in their study reported using at least one CAM method for the past 6 months. The most common methods used by participants were prayer (45\%), spiritual healing by others (34\%), massage therapy (31\%), and relaxation (23\%), often multiple methods were employed by the same caregiver [11]. Megavitamins (19\%), herbal products (11\%), heat application, and warm baths were also frequently used [11]. One hundred percent of caregivers who completed their questionnaires responded with interest in using some types of complementary therapies for their kids to manage pain in the future, most notably: $95.2 \%$ wanted to use prayer, massage therapy (79.4\%), relaxation techniques (73.0\%), spiritual healing by others (71.0\%), music (61.3\%), acupuncture (11.3\%), and hypnosis (12.7\%) [11]. Post-White et al. [12] report similar findings, $77.8 \%$ of their sickle cell patients using CAM used prayer, followed by massage $(44.4 \%)$ and vitamins (22.2\%). Others less prevalent CAM methods were supplements $(11.1 \%)$, relaxation (22.2\%), music (33.3\%), imagery (11.1\%), herbals (22.2\%), and aromatherapy (11.1\%) [12].

More specifically, Dampier et al. [7] evaluated the use of CAM in pediatric SCD patients and reported CAM methods based on passive and active CAM. Initially, they report that CAM was used on $84.6 \%$ of days with sickle pain, alongside analgesics on most of these days [7]. Most commonly, a combination of activities used involved passive distraction activities (sleeping, watching TV, talking with friends) rather or active (relaxation/meditation/self-hypnosis, use of heat and massage) [7]. The most common were passive distraction activities (sleeping used on $21.5 \%$ of SCD pain days, watching TV used on $24 \%$ of SCD pain days, talking to friends used on $16.7 \%$ of SCD pain days) [7]. Out of the active methods, the frequency of use on sickle cell pain days was: heat application (hot bath, pad) (10.9\% of pain days), massage (8.0\%), prayer (7.2\%), relaxation/meditation/self-hypnosis (11.7\%) [7].

Finding similar results that CAM was widely used amongst SCD patients, Powers et al. [6], however, report that breathing was the most often used CAM as a means of active coping (45.7\% of the time), followed by relaxation (29.3\%), distraction (38.5\%), and positive self-talk (28.7\%). Gil et al. [13], found that with higher pain levels, children with SCD practiced coping skills (deep breathing relaxation, pleasant imagery, calming self-talk) more frequently, and were less likely to have major healthcare encounters and negative thinking. They were also more likely to remain involved in school and in household activities.

In contrast to the above findings of their North American counterparts, Oshikoya et al. [14] investigated the use of CAM for children with chronic health conditions, including SCD, report that out of their study participants, only $36 \%$ of sickle cell anemia (SCA) patients used CAM. Biological products were most frequently used as CAM (58\%), followed by alternative medical systems (27\%) [14]. Only $3.4 \%$ of SCA patients used massage, the only manipulation and touch CAM therapy used [14]. Fifty-two percent of patients were also using more than one biological therapy at a time [14]. Most patients used CAM daily, a few used it weekly or occasionally [14]. 


\subsection{Factors Associated with the Use of CAM}

CAM practices have gained more popularity in the last few decades, especially among SCD patients. However, it is important to explore the factors associated with CAM use, whether they are motivating or limiting its use, to better understand the applicability of CAM.

Sibinga et al. [10] found that CAM use was associated with age, number of hospitalizations, perceived impact of SCD on life, respondent education, pain crises, SCD severity, and increased family use. They found that the mean age for CAM users was 9.7 years old as opposed to 6.4 years old for non-users $(p<0.02)$ [10]. CAM was almost twice as common in patients with multiple hospitalizations in the previous 12 months [10]. It was positively associated with higher scores on an SCD Severity Scale $(p=0.03)[10]$. A higher perceived impact of SCD on the child's life was positively associated with CAM use $(p=0.05)$ [10]. Specifically, the use of lifestyle/mind-body therapies were associated with SCD severity, higher scores on the SCD severity scale were associated with greater use of relaxation techniques, (OR 1.23, $p<0.05)$ [10]. Interestingly, they found that patients with pain crises in preceding years were more likely to use bioenergetics therapies $(p<0.05)$ [10]. This finding is similar to Yoon et al. [11] who discovered that CAM use was significantly higher in children taking two or more analgesics for pain control compared to kids taking none or one analgesic $(p=0.047)[11]$.

Beyond medical associations, Sibinga et al. [10] found that higher respondent level of education $(p<0.05)$ and increased family CAM use in general, and herb and prayer use, in particular, were associated with CAM use for the SCD child (all $p<0.01$ ). In their sample, CAM use was not associated with family income [10]. Gil et al. [13] report similar results, a higher level of education in parents predicted more frequent daily coping practices in children $(p<0.01)$. However, they found that age predicted the frequency with which children practiced their coping skills $(p<0.01)$, with younger children practicing them more frequently. They also found children who experienced more chronic disease complications were less likely to practice their coping skills $(p<0.01)[13]$.

Interestingly, they found that disease type predicted frequency of coping practice $(p<0.05)$, children with Hemoglobin SC disease practiced strategies more than those with SS anemia (HbSS) [13]. Otherwise, they specifically found that girls practice CAM more frequently each day than boys $(p<0.01)$, and children whose reporting parent was divorced practiced most frequently, followed by children of married parents, and then by children of never-married parents $(p<.01)$ [13].

Dampier et al. [7] found congruent results as Gil et al. [13]. Female subjects tended to use sleeping/napping, relaxation/meditation/self-hypnosis, and massage more frequently than did male subjects ( $p=0.001,0.003,0.047$ respectively), the mean number of activities used by females (2.2 methods) was $70 \%$ greater than for males ( 1.3 methods, $P=0.003$ ) [7]. They also found that older patients tended to use praying less than younger patients $(p=0.043)$ [7]. However, they did also find similar to Sibinga et al. [10] that disease severity was associated with CAM use [7]. Frequency of use of sleeping/napping, massage, heat, and relaxation/meditation/ self-hypnosis, increased with increasing pain intensity (significant 1.5-2-fold increase in expected frequency as pain intensity increased, $p<0.05$ for all) [7]. Active and passive strategies increased with increasing pain intensity, at higher intensity more active strategies were used [7]. During home management of SCD pain, most children utilized passive approaches compared to only $11.7 \%$ who used more active modalities to alleviate their pain [7]. The use of active modalities 
increased drastically with an increase in pain intensity, and patients reported using active strategies over $85 \%$ of the time on their "worst pain" days [7]. For every unit increase in pain intensity, the expected number of activities used increased by $3.5 \%(p=0.04)$ [7].

Sanchez et al. [9] also similarly found that child CAM use significantly correlated with parents' use of CAM ( $r=0.69, p<0.001$ ). In contrast to the above studies, they showed no significant difference in CAM use between male and female children (22 and 13\% $p=0.29$ ) [9]. However, they explored more socioeconomic aspects of CAM use as well. They found that of those who used CAM, $90 \%$ spent less than $\$ 100$ on CAM total [9]. They also reported that white children had a lower prevalence of non-prayer CAM use $(22 \%)$ than minority children $(29 \% p<0.001)$ [9]. However, the use of different categories of CAM (mind-body practices and natural products) was not significantly related to patient diagnosis [9]. They uncovered the participants' main reasons for using CAM in SCD patients were to provide hope (25\%) and improve quality of life (50\%) [9]. Finally, as per Sanchez et al. [9], 95.6\% of patients who did not use any form of CAM expressed that lack of information was an important contributing factor in their decision [9]. However, most of them were interested in discussing the use of CAM with their healthcare provider [9].

Post-White et al. [12] expanded on the most common reasons for using CAM in SCD children. They reported the following reasons and their frequency for caregivers to employ CAM: because it is helpful to the parent (44.4\%), might help fight illness $(55.6 \%)$, recommended to the parent (33.3\%), to feel more hopeful (55.6\%), to cope with emotional effects $(44.4 \%)$, to manage side effects (11.1\%), to have more participation in child's medical care (11.1\%), standard therapy does not offer child enough (33.3\%), dissatisfied with standard care (22.2\%), and child's health has changed for worse (11.1\%) [12]. However, this study, once again found that parents who used some type of CAM therapy were more likely to use CAM for their child $(p<0.0001)$, and that parent use was the only significant predictive factor of child's use of CAM $(p<0.0001)$ [12]. In contrast to the studies above, they did not find that child age, gender, and race and parental age, income, and education had any significant influence on CAM use [12].

The reasons that Post-White et al. [12] found for using CAM are supported by the findings of Oshikoya et al. [14]. They also discovered that relatives, friends, and neighbors influenced parents to use CAM for children [14]. Also, parents used CAM to treat or cure their children, improve physical conditions, relieve symptoms of illness [14]. Reasonably, a significant number of sickle cell anemia patients used CAM to improve body immunity $(p<0.001)$ [14]. Reasons stated by participants of the study for discontinuing CAM were adverse reactions (fever, diarrhea, vomiting in SCA patients), dissatisfactions, and lack of benefits [14].

Studies have not yet reported a clear association between income and CAM use [12]. Sanchez et al. [9] postulated that non-prayer CAM use was limited in Mississippi due to financial constraints and limited insurance coverage [9].

Table 2 CAM participation percentages as reported across the literature.

\begin{tabular}{lllllll}
\hline & Dampier & Sibinga & Yoon & Post-White & Sanchez & Oshikoya \\
& 2004 & 2006 & 2006 & 2009 & 2015 & 2008 \\
\hline Overall Use & $84.60 \%$ & $54 \%$ & $70 \%$ & $47.40 \%$ & $63 \%$ & $36 \%$ \\
Prayer & & $42 \%$ & $45 \%$ & $77.80 \%$ & $60 \%$ & $33 \%$ \\
Used spiritual healing & & $11 \%$ & $34 \%$ & & & $27 \%$
\end{tabular}




\begin{tabular}{|c|c|c|c|c|c|}
\hline Energy healing & $2 \%$ & & & & \\
\hline $\begin{array}{l}\text { Lifestyle/mind-body } \\
\text { techniques (exercise, diet, } \\
\text { and/or imagery techniques) }\end{array}$ & $28 \%$ & & $11.10 \%$ & & \\
\hline $\begin{array}{l}\text { Biochemical therapies (herbs, } \\
\text { megavitamins, and/or folk } \\
\text { remedies) }\end{array}$ & $12 \%$ & & $33.30 \%$ & $10 \%$ & $58 \%$ \\
\hline Massage therapy & $5 \%$ & $31 \%$ & $44.40 \%$ & $7.50 \%$ & $11 \%$ \\
\hline Relaxation & & & $22.20 \%$ & & \\
\hline Music & & & $33.30 \%$ & & \\
\hline Aromatherapy & & & $11.10 \%$ & & \\
\hline
\end{tabular}

\subsection{Effectiveness of CAM}

Since SCD targets multiple systems, it demands a multidisciplinary approach to treatment. CAM offers a promising avenue of alternative therapy for SCD pain. Given the increasing use of CAM in the past few decades, this review presents an excellent opportunity to explore the feasibility and efficacy of CAM in children with SCD. In this section, we report the findings on the effectiveness of CAM on improving patient lives.

Despite recent improvements in survival rates among children with SCD, pain remains the most common complication contributing to the decrease in quality of life for these patients. Managing chronic and acute pain (VOC) with conventional treatments has had limited success. Any interventions that alleviate pain and decrease analgesics use are well received by patients. Biofeedback, yoga, guided imagery, virtual reality, massage therapy, acupuncture show promising results in reducing the pain in this population [15-20]. These techniques may be used alone or in combination with conventional treatments.

In one study, patients were trained to use biofeedback-assisted relaxation training (BART) to help with pain control [18]. One-hour BART training sessions increased peripheral body temperature (reflecting relaxation) immediately after training, and when measured 6 weeks later (immediately after training mean PBT change $=2.20^{\circ} \mathrm{C}$; SD $=2.08^{\circ} \mathrm{C}$, and 6 weeks after training mean PBT change $=2.24^{\circ} \mathrm{C} ; \mathrm{SD}=2.53^{\circ} \mathrm{C}$ ) [18]. Decreased pain frequency in children with SCD was also reported after BART by all the participants (3.1 vs 1.78 pain days/week pre-, and post-BART, respectively) [18]. Caregivers and participants both found BART to be an acceptable intervention for SCD pain [18].

The study by Moody et al. [19] reported that one yoga session significantly reduced the mean pain score for pediatric SCD patients experiencing VOC $(-0.6 \pm 0.96$ vs. $0.0 \pm 1.37 ; \mathrm{P}=0.029)(\mathrm{N}=$ 73) [19]. As yoga skills are likely to improve with practice, patients may achieve greater benefits with time [19]. Nevertheless, there was no significant difference in the mean anxiety score, total intravenous opioid use, or length of stay in hospital between patient groups that practice yoga and those who did not [19].

Lemanek et al. [17] showed that youth who received nightly massages for 30 days, provided by trained caregivers, reported decreased pain $(\mathrm{N}=34)$. Children and adolescents and their parents were assigned to massage therapy or control groups, parents were then trained in massage in 
their homes with instructions to give nightly massages [17]. Participants completed measures of anxiety, functional status, pain intensity, medication use, and service utilization [17]. Interestingly, caregivers in the massage group scored significantly less in] anxiety measures (STAI state anxiety scale) $(p<0.0001)$ than the control group [17]. However, ratings of functional status for the massage therapy group were higher compared to the control group $(p=0.0001)$ [17]. Youth in the massage therapy group rated their levels of depression, anxiety, and pain significantly lower than those in the control group ( $p=0.05, p=0.01, p=0.05$, respectively) [17]. Health service utilization rates were not significantly different for the frequency of ED visits, hospitalizations, or the number of days hospitalized [17].

Gil et al. [13] evaluated the effects of daily coping practices with audiotaped instructions for relaxation/imagery skills on SCD patients [13]. Forty-six African American children with SCD were randomly assigned to either the coping skills condition group or the standard care control condition group [13]. They were both asked to practice daily coping with audiotaped instructions for relaxation and/or imagery skills [13]. Children were trained to use three coping strategies: deep breathing relaxation/pleasant imagery and calming self-talk [13]. Overall completion rate was 55\% (completed diaries on 1071 days/1937 possible days) [13]. At 1 month follow-up, children in coping skills condition reported that they took a more active approach to manage their pain than those in control [13]. When children practiced their skills on a day with pain, they were significantly less likely to have a major health care contact such as an ER or clinic visit or hospital admission $(p<0.01)$ [13]. They were also significantly more likely to remain involved in school activities $(p<0.01)[13]$.

Virtual Reality (VR) is a promising candidate as synergistic with opioids for pain reduction in pediatric patients with SCD [21]. Previous studies demonstrated that the combined effect of opioids and virtual reality (VR) decreased the subjective pain score in healthy individuals more than opioids alone [21]. Agrawal et al. [15] showed that a single 15-minute VR therapy session (KindVR Aqua software) statistically decreased VOC pain intensity, the number of affected body areas, and qualitative pain measures [15]. With a sample of 30 patients, they provided VR therapy in hospitalized patients for VOC [15]. Patients had significant improvements in all aspects of the adolescent pediatric pain tool (APPT) after 15-min VR session [15]. Median pain intensity decreased from 7.3 to 5.8 ( $p<0.001$ ), and the median number of affected body areas decreased from 3.0 to 2.0 post-VR $(p<0.001$ ) [15]. Furthermore, qualitatively, the mean percentage of adjectives used to describe pain (a certain number of adjectives that the participants circled out of 67 possible descriptors) also decreased from $20.1 \%$ to $12.7 \%(p<0.001)$ [15].

Guided imagery has also been shown to significantly reduce pain intensity in children with SCD [16]. Dobson et al. [16] evaluated the use of guided imagery to manage pain in youth with SCD. Participants reported using the guided imagery following training anywhere between 0 to 23 days in the post-intervention month (average of 7.75 days, $N=20$ ) [16]. There was a modest and inverse correlation between pre-intervention self-efficacy scores, and frequency of guided imagery use $(r=-0.481, P=0.03)$ [16]. They showed that after receiving training on using guidedimagery and its subsequent use, children reported fewer pain episodes per month and reduced pain intensity (5.6 pain episodes pre-intervention SD $=3.3$ vs 2.5 pain episodes SD $=4.1$ postintervention $p=0.003$, and a pain score of $2.4 / 6 \mathrm{SD}=1.2$ pre-intervention vs $0.7 / 6 \mathrm{SD}=1.2$ postintervention $p=0.00$ ) [16]. At the end of the study, frequency of use correlated positively at a 
trend level with change in scores for self-efficacy, the greatest increase in self-efficacy scores was seen in those children who used guided imagery more often $(r=0.43, p=0.06)$ [16].

Acupuncture is often a therapy that adults use for various reasons, including pain. However, some parents are hesitant to use acupuncture for their children. Contrary to popular belief, children tolerate acupuncture well and find it helpful [22]. Mahmood et al. [20] investigated acupuncture as a pain management tool for kids with SCD. Out of 12 participants, 11/12 (92\%) tolerated acupuncture well and 7 patients received $>1$ sessions of acupuncture, demonstrating an ongoing interest in therapy [20]. One person got superficial scratches from intradermal stick-on needles and declined further therapy, however, 100\% of 11 felt it was a good experience with $73 \%$ specifically expressing improvement in pain/feeling good after the session [20]. Among the 15 sessions where pre- and post-acupuncture pain scores were documented, the mean preacupuncture pain score was $6.17 \pm 2.73$ and the mean post acupuncture was $5.23 \pm 2.46$ (mean \pm SD) [20]. The average difference in pain scores from pre- to post-acupuncture sessions was 0.933 with a standard deviation of 1.03 and, after accounting for repeated measures per person, was statistically significantly different from $0(p<0.000)[20]$. 
OBM Integrative and Complementary Medicine 2021; 6(2), doi:10.21926/obm.icm.2102013

Table 3 Factors associated with CAM use across the literature.

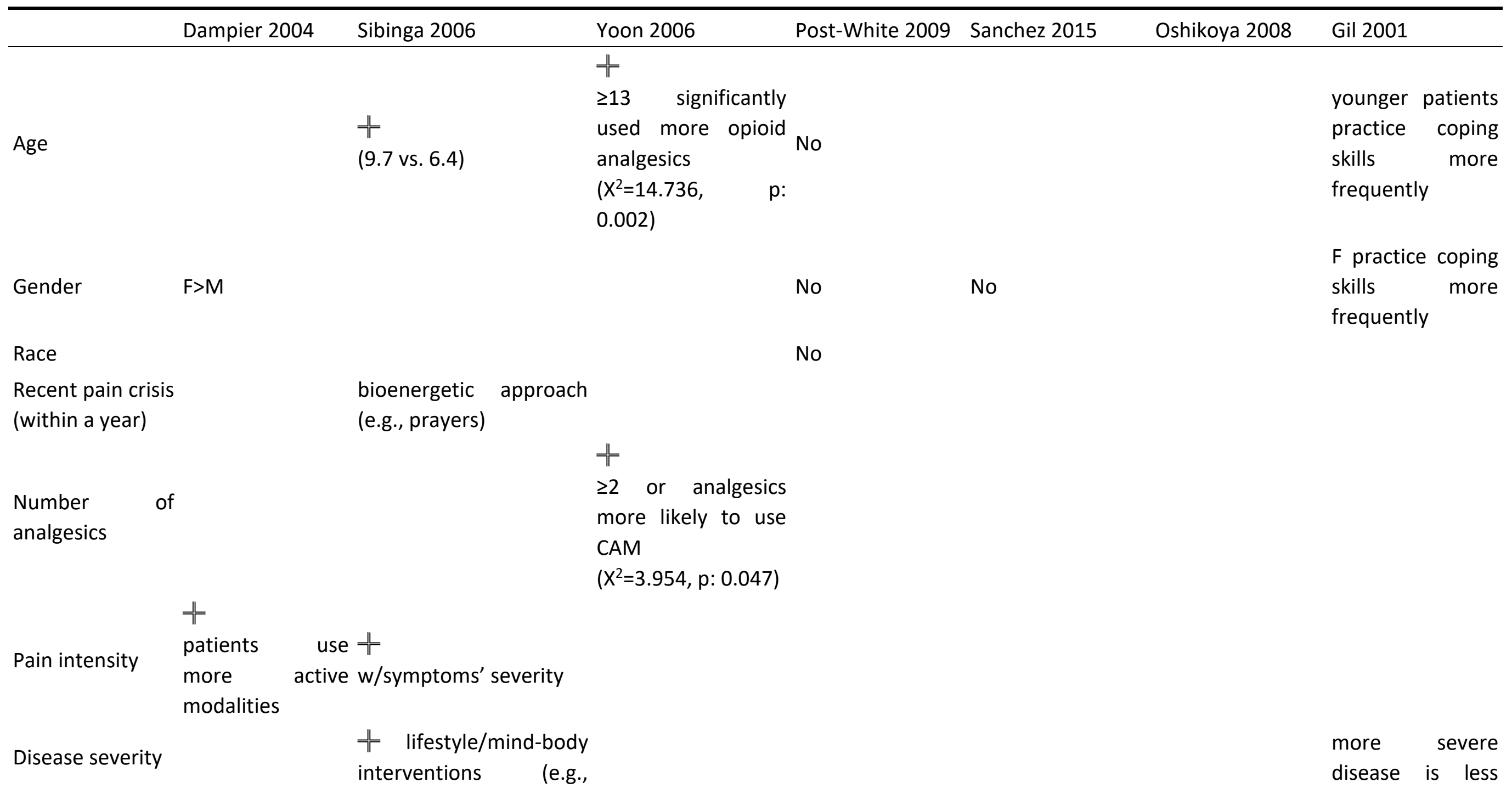




\section{relaxation)}

Parental age

\section{Parental use}

\section{Parental}

education Level

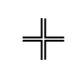

Parental income
No

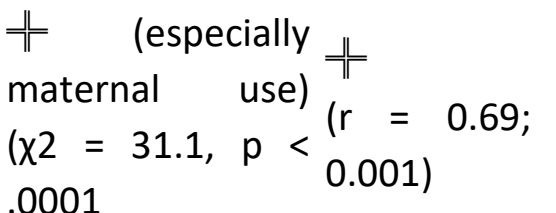

0001

No

\section{Non-prayer CAM}

use was limited in

No

financial
Mississippi due to

likely to

use

coping skills

Relatives, friends,

and neighbors

influenced

parents to use

CAM for children

(79\%)

$\Perp$

W educated, working, divorced parents coping skills

constraints and

limited insurance

coverage 


\section{Discussion}

This narrative review examined recent literature from 2000 to 2020 on the prevalence and factors associated with CAM use among children with SCD, as well as the effectiveness of CAM on different aspects of SCD such as pain, depression, anxiety, hospitalizations/ED visits, daily function, and analgesic use. Regarding the prevalence of CAM use, our review found that while most patients with SCD pain use some type of CAM (average of $63.8 \%$ in the US-based literature), the most widely used type is prayer, followed by spiritual healing and biochemical therapies. While the patients who use these therapies tend to be older children, coping skills, on the other hand, were mainly used by younger patients [13]. These results highlight the importance of taking into consideration the widespread use of CAM in the majority of SCD patients with pain crises. It also serves as a guide for hospitals, clinics and healthcare providers as this study shows the types of CAM most commonly used and the current scientific evidence available about them. Finally, by discussing each type of CAM, this study helps determine what types of services hospitals can make available for patients.

The main factors associated with CAM use were parental use, especially maternal use $[9,12]$. Reasons for this were related to the ability of CAM to help fight illness, feel more helpful in addressing pain, helpful to caregivers, and cope with the emotional effects of SCD pain [12]. Barriers to CAM use were related to the lack of information about CAM, financial constraints, and limited insurance coverage for CAM services [9]. This is particularly important as these barriers show possible future interventions that would improve the widespread access to CAM services. For example, some insurance companies may cover very few CAM therapies (e.g., chiropractic medicine and acupuncture) but not others (BART, VR, and guided imagery); thus leaving patients and families to pay for other safe and effective therapies out of pocket thereby increasing their financial burden.

Biofeedback, yoga, guided imagery, VR, massage therapy, acupuncture showed promising results in reducing SCD pain $[15-17,19,20]$. In the case of anxiety, massage also was shown to provide adequate relief [17]. Alternatively, BART, yoga, and VR have shown to be adequate adjunct therapies $[15,18,19]$. Furthermore, patients felt the benefits of learning daily coping strategies even after a brief training [13]. They were also able to recall and implement the strategies learned at later points in time when their pain was at its highest point. This may have major implications for the ability of these behavioral and psychological interventions to ease the burden on ED overuse and hospital readmissions specific to VOC; however, this needs to be further studied. Also, more research is needed to assess the usefulness of CAM as a tool for reducing and controlling the devastating and overwhelming opioid epidemic in the US.

The limitations of this narrative review lie in the limited number of studies available that addressed the prevalence, factors related to use and effectiveness of CAM for the management of SCD pain in children, as well as the factors related to discontinuation of CAM use. As the use of CAM in SCD increases, more studies that address the risks and benefits of each CAM method are needed. Current evidence highlights the need to conduct more long-term studies addressing the research gaps, barriers, and specific methods that directly affect the patient's quality of life. The use of objective and biological markers would also allow us to strengthen the validity of these studies. Finally, as novel technologies (e.g., apps, VR) and social media continue to have a greater 
influence on the daily learning and recreational activities of children and adolescents, further studies are needed to assess their effects on SCD pain and related symptoms.

\section{Conclusion}

SCD is a chronic and progressive disease with devastating physiological, psychological, and psychosocial effects on the patients. Pain is the most common manifestation of SCD. The sudden onset, and intermittent nature of SCD pain poses a challenge in the management of this disease. In recent decades, CAM usage has seen an upward trend in the pediatric population which demands more research to examine the feasibility and efficacy of these methods. CAM introduces a biopsychosocial model of pain treatment that can be adopted at home and in inpatient settings. Literature has shown that CAM use holds potential in providing pain relief and these interventions could be integrated into traditional treatment plans, with further research on a larger scale investigating efficacy and long-term effects. Pediatric patients with SCD may benefit from these practices solely or in conjugation with conventional medicine, as CAM holds great promise in improving the quality of life of children with SCD.

\section{Acronyms}

SCD: Sickle cell disease

CVA: cerebrovascular accidents

ACS: acute chest syndrome

CAM: complementary and alternative medicine

TV: Television

BART: Biofeedback-assisted relaxation training

ED: Emergency Department

$\mathrm{NCClH}$ : National Center for Complementary and Integrative Health

\section{Author Contributions}

Kusum Viswanathan (KV) and Mohammed Alsabri (MA) proposed the project, contributed to the conception, formulation, and drafting of the article, and reviewed and revised the manuscript. Fiorella Castillo (FC), Paridhi Ghai (Gh), Maryam Hemmat (MH); reviewed the literature, analysed data, and drafted the manuscript. Paridhi Ghai (GH) and Fiorella Castillo (FC) contributed equally to the drafting of the article. Prof. Kusum Viswanathan (KV) supervised the project and mentored the team, she is the corresponding and senior author. All authors read and approved the final manuscript.

\section{Competing Interests}

The authors have declared that no competing interests exist.

\section{References}

1. Regional Committee for Africa. Sickle-cell disease: A strategy for the WHO African Region [Internet]. Genève: World Health Organization; 2011. Available from: 


\section{https://apps.who.int/iris/handle/10665/1682.}

2. Edwards CL, Scales MT, Loughlin C, Bennett GG, Harris-Peterson S, De Castro LM, et al. A brief review of the pathophysiology, associated pain, and psychosocial issues in sickle cell disease. Int J Behav Med. 2005; 12: 171-179.

3. Claster S, Vichinsky EP. Managing sickle cell disease. BMJ. 2003; 327: 1151-1155.

4. Ballas SK, Gupta K, Adams-Graves P. Sickle cell pain: A critical reappraisal. Blood. 2012; 120: 3647-3656.

5. Williams H, Tanabe P. Sickle cell disease: A review of nonpharmacological approaches for pain. J Pain Symptom Manage. 2016; 51: 163-177.

6. Powers SW, Mitchell MJ, Graumlich SE, Byars KC, Kalinyak KA. Longitudinal assessment of pain, coping, and daily functioning in children with sickle cell disease receiving pain management skills training. J Clin Psychol Med Settings. 2002; 9: 109-119.

7. Dampier C, Ely E, Eggleston B, Brodecki D, O'Neal P. Physical and cognitive-behavioral activities used in the home management of sickle pain: A daily diary study in children and adolescents. Pediatr Blood Cancer. 2004; 43: 674-678.

8. Stinson J, Naser B. Pain management in children with sickle cell disease. Paediatr Drugs. 2003; 5: 229-241.

9. Sanchez HC, Karlson CW, Hsu JH, Ostrenga A, Gordon C. Complementary and alternative medicine use in pediatric hematology/oncology patients at the University of Mississippi Medical Center. J Altern Complement Med. 2015; 21: 660-666.

10. Sibinga EM, Shindell DL, Casella JF, Duggan AK, Wilson MH. Pediatric patients with sickle cell disease: Use of complementary and alternative therapies. J Altern Complement Med. 2006; 12: 291-298.

11. Yoon SL, Black S. Comprehensive, integrative management of pain for patients with sickle-cell disease. J Altern Complement Med. 2006; 12: 995-1001.

12. Post-White J, Fitzgerald M, Hageness S, Sencer SF. Complementary and alternative medicine use in children with cancer and general and specialty pediatrics. J Pediatr Oncol Nurs. 2009; 26: 7-15.

13. Gil KM, Anthony KK, Carson JW, Redding-Lallinger R, Daeschner CW, Ware RE. Daily coping practice predicts treatment effects in children with sickle cell disease. J Pediatr Psychol. 2001; 26: 163-173.

14. Oshikoya KA, Senbanjo IO, Njokanma OF, Soipe A. Use of complementary and alternative medicines for children with chronic health conditions in Lagos, Nigeria. BMC Complement Altern Med. 2008; 8: 66.

15. Agrawal AK, Robertson S, Litwin L, Tringale E, Treadwell M, Hoppe C, et al. Virtual reality as complementary pain therapy in hospitalized patients with sickle cell disease. Pediatr Blood Cancer. 2019; 66: e27525.

16. Dobson $\mathrm{CE}$, Byrne MW. Using guided imagery to manage pain in young children with sickle cell disease. Am J Nurs. 2014; 114: 26-36.

17. Lemanek KL, Ranalli M, Lukens $C$. A randomized controlled trial of massage therapy in children with sickle cell disease. J Pediatr Psychol. 2009;34: 1091-1096.

18. Myrvik MP, Campbell AD, Butcher JL. Single-session biofeedback-assisted relaxation training in children with sickle cell disease. J Pediatr Hematol Oncol. 2012; 34: 340-343.

19. Moody K, Abrahams B, Baker R, Santizo R, Manwani D, Carullo V, et al. A randomized trial of 
yoga for children hospitalized with sickle cell vaso-occlusive crisis. J Pain Symptom Manage. 2017; 53: 1026-1034.

20. Mahmood LA, Reece-Stremtan S, Idiokitas R, Martin B, Margulies S, Hardy SJ, et al. Acupuncture for pain management in children with sickle cell disease. Complement Ther Med. 2020; 49: 102287.

21. Hoffman HG, Richards TL, Van Oostrom T, Coda BA, Jensen MP, Blough DK, et al. The analgesic effects of opioids and immersive virtual reality distraction: Evidence from subjective and functional brain imaging assessments. Anesth Analg. 2007; 105: 1776-1783.

22. Kemper KJ, Sarah R, LicAc MP, Silver-Highfield E, Xiarhos E, Barnes L, et al. On pins and needles? Pediatric pain patients' experience with acupuncture. Pediatrics. 2000; 105: 941-947.

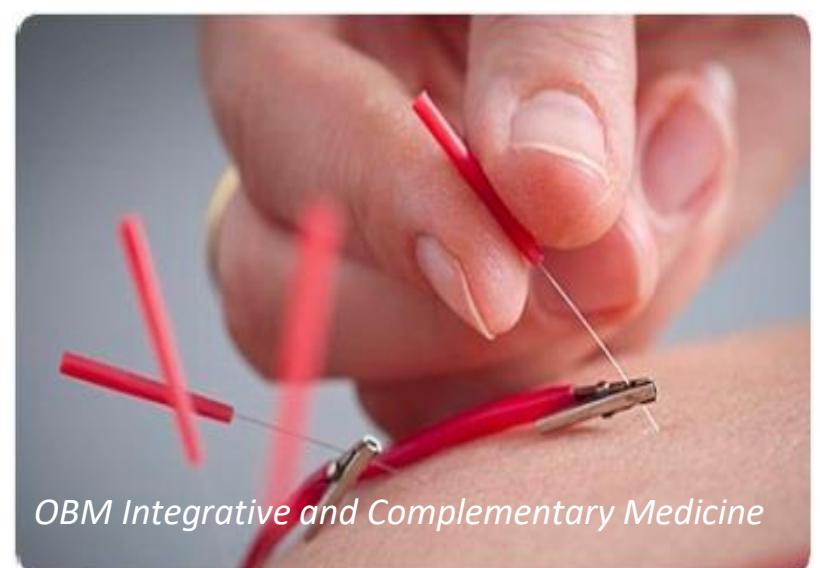

Enjoy OBM Integrative and Complementary Medicine by:

1. Submitting a manuscript

2. Joining in volunteer reviewer bank

3. Joining Editorial Board

4. Guest editing a special issue

For more details, please visit: http://www.lidsen.com/journals/icm 